\title{
Determinación de monóxido de carbono (Co) como factor de riesgo laboral en estaciones de servicio de combustible
}

\author{
Determination of carbon monoxide (CO) \\ as an occupational \\ risk factor at fuel service stations
}

Determinação do monóxido de carbono (CO) como fator de risco laboral nos posto de combustível

\author{
Isidro Andrés Tejedor Cassiani ${ }^{1} \&$ Nini Johana Mena² \\ 'Biólogo, Magister en Toxicología. \\ ${ }^{2}$ Administradora en Salud Ocupacional. \\ 1, 2Programa de Administración en Salud Ocupacional. \\ Corporación Universitaria Minuto de Dios, Pitalito. Huila, Colombia.
}

1isidro.tejedor@uniminuto.edu, ${ }^{2}$ nmenaarbole@uniminuto.edu.co

\section{Resumen}

El propósito de la investigación fue la determinación del $\mathrm{CO}$ como factor de riesgo laboral en las estaciones de servicio del municipio de Pitalito, Huila, Colombia, donde se realizaron mediciones de las concentraciones de $\mathrm{CO}$ en los ambientes laborales de 9 estaciones de servicio de gasolina, mediante el monitor de gases portátil GX 2009, marca RKI que permite medir concentración de Oxigeno, CO, $\mathrm{H}_{2} \mathrm{~S}$ e hidrocarburos alifáticos. Estas mediciones se realizaron cada 15 minutos en el transcurso de 2 horas en la mañana, al medio día, la tarde y la noche, repitiendo el procedimiento durante 3 jornadas de 8 horas laborales. En la primera estación se obtuvo un promedio de $37,20 \mathrm{ppm}$ de CO con una desviación estándar de 19,47 (37,20 $\pm 19,47)$, en la segunda se obtuvo $48,82 \mathrm{ppm}$ de $\mathrm{CO}$ y una desviación estándar de 25,82 $(48,82 \pm 25,82)$, en la tercera $(28,12 \pm 24,15)$, en la cuarta $(37,05 \pm 21,44)$,en la quinta $(10,77 \pm 12,56)$, la sexta $(30,47 \pm 25,05)$, en la séptima $(43,53 \pm 30,09)$, en la octava $(42,55 \pm 30,96)$, en la novena $(27,72 \pm 20,99)$ y por último la media de las concentraciones de CO durante una jornada laboral en las estaciones de servicio corresponde a $34,02 \mathrm{ppm}$. Se concluye que las concentraciones de CO no siguen un patrón de relación directamente proporcional con los síntomas manifestados por los trabajadores; pero muchas de están alteraciones pueden estar directamente relacionadas con el tiempo de exposición al CO.

Palabras clave: monóxido de Carbono, factor de riesgo, estaciones de servicio, Pitalito

\section{Abstract}

The purpose of the research was the determination of $\mathrm{CO}$ as a risk factor for workers in the service stations of the municipality of Pitalito, Huila, Colombia, where measurements were made of the concentration 
of $\mathrm{CO}$ in the work environments of 9 service stations of petrol, through the gas monitor laptop GX 2009, brand RKI that allows to measure concentration of oxygen, $\mathrm{CO}, \mathrm{H} 2 \mathrm{~S}$ and aliphatic hydrocarbons. These measurements were carried out every 15 minutes during 2 hours in the morning, at mid-day, evening and night, repeating the procedure during the 3 days of 8 working hours. In the first station was obtained an average of $\$ 45.32 \mathrm{ppm}$ CO with a standard deviation of $19,47(37,20 \pm 19,47)$, in the second was obtained 48,82ppm CO and a standard deviation of 25,82 $(48,82 \pm 25,82)$, in the third $(28.12 \pm 24.15)$, in the fourth $(37.05 \pm 21,44)$, in the fifth $(10.77 \pm 12.56)$, the sixth (- 30.47 \pm 25.05$)$, in the seventh $(43,53 \pm 30,09)$, in the eighth $(42,55 \pm 30,96)$, in the ninth $(27,72 \pm 20,99)$ and finally the average concentrations of $\mathrm{CO}$ during a labor day in service stations corresponds to 34,02ppm. It is concluded that the concentrations of $\mathrm{CO}$ do not follow a pattern of directly proportional relationship with symptoms manifested by the workers; but many are alterations can be directly related to the time of exposure to the $\mathrm{CO}$.

Key-words: Carbon monoxide, risk factor, service stations, Pitalito

\section{Resumo}

O propósito do estudo é a determinação do $\mathrm{CO}$ como fator de risco laboral nos postos de combustível de
Pitalito, Huila, onde foram feitas medidas de concentrações de $\mathrm{CO}$ nos ambientes de trabalho de 9 pontos de combustível do local usando-se o monitor de gases portátil GX 2009, marca RKI que permite medir a concentração de Oxigênio, $\mathrm{CO}, \mathrm{H}_{2} \mathrm{~S}$ e hidrocarbonetos alifáticos. Estas medições foram feitas cada 15 minutos ao longo de 2 horas no horário da manhã, meio dia, tarde e noite, repetindo o procedimento em 3 expedientes de 8 horas. No primeiro posto foi obtida uma média de $37,20 \mathrm{ppm}$ de CO com um desvio padrão de 19,47 (37,20 \pm $19,47)$, no segundo posto encontrou-se 48,82 ppm de CO e um desvio padrão de 25,82 (48,82 \pm $25,82)$, no terceiro posto $(28,12 \pm 24,15)$, no quarto posto $(37,05 \pm 21,44)$, no quinto posto $(10,77 \pm$ $12,56)$, no sexto posto $(30,47 \pm 25,05)$, no sétimo posto $(43,53 \pm 30,09)$, no oitavo posto $(42,55 \pm$ $30,96)$, no noveno $(27,72 \pm 20,99)$ e finalmente a média das concentrações de $\mathrm{CO}$ durante um dia de expediente nos postos de combustível corresponde a 34,02 ppm. Conclui-se que as concentrações de CO não seguem um padrão de relação diretamente proporcional com os sintomas observados nos trabalhadores, mesmo assim, muitas das alterações podem estar diretamente relacionadas com o tempo de exposição ao CO.

Palavras-chave: Monóxido de Carbono, Fator de Risco, Postos de combustível, Pitalito

\section{Introducción}

En el presente siglo, diversos estudios han puesto en manifiesto, que la intoxicación por monóxido de carbono (CO) es la primera causa de muerte accidental en la sociedad actual, la cual podría ser considerada como potencialmente letal, cuando se relaciona en un contexto de intento de suicidio; aunque en Europa y muchas partes del mundo, suele ser accidental con mucha frecuencia (Hampson, 2016). Esta situación, permite pensar en este tipo de intoxicación, cuando el xenobiótico relacionado es desconocido y el diagnóstico de la misma es algo dudoso (Donati et al., 2005).
Algunos de las propiedades fisicoquímicas de esta sustancia, permiten definir al $\mathrm{CO}$ como un gas inodoro, incoloro, insípido, no irritante, inflamable y potencialmente explosivo, que en ocasiones se comporta como un compuesto relativamente inerte, por lo que necesita la interacción con algunos contaminantes como el ozono y oxígeno para aumentar su letalidad (Guzmán, 2012); pero en realidad, se difunde con gran rapidez en el medio ambiente y en el organismo se une con el grupo hemo de moléculas de hemoglobina al interior de los glóbulos rojos, para formar compuestos de carboxihemoglobina, 
desplazando al oxígeno y evitando su transporte hasta las células del organismo, haciendo que una persona muera por hipoxia (Hampson \& Bodwin, 2013; Castillo \& Casan, 2006). En algunos casos, en el metabolismo de proteínas como hemoglobina, citocromos, catalasas, mioglobina y peroxidasas se produce fisiológicamente hasta $10 \mathrm{ml}$ de $\mathrm{CO}$ en un día; no obstante, la fuente primaria de $\mathrm{CO}$, ocurre a partir de fuentes exógenas (Sandilands \& Bateman, 2016). En la mayoría de los casos, el CO es el producto de la combustión incompleta de hidrocarburos como la gasolina durante el funcionamiento de motores de vehículos, puede producirse a partir de incendios de bosques, hace parte de las emisiones de los volcanes durante sus erupciones (Romero et al., 2006). Por consiguiente, la exposición a este toxico es de mayor preocupación desde el punto de vista accidental, doméstico y ocupacional; aunque la predominación, sigue presentándose en ambientes relacionados con calefacción defectuosos y los gases emitidos por los automóviles.

La exposición al CO pueden conllevar a la manifestación de cefaleas, vértigos, debilidad muscular, náuseas o vómitos, perdida del conocimiento, alteraciones visuales, dolores torácicos y dolor abdominal (Fleta et al., 2005); (Oliu et al., 2010). A raíz de las diferentes complicaciones que puede desarrollar las personas expuestas, la Organización Mundial para la Salud (OMS) ha definido algunos parámetros relacionados con estas concentraciones. Por tal motivo, un trabajador no puede exceder a una exposición de 10ppm de CO durante una jornada laboral de 8 horas continuas en un ambiente abierto, ni $90 \mathrm{ppm}$ durante 15 minutos en la misma jornada en las mismas condiciones (Donati et al., 2005); (Vargas, 2006).

La exposición al CO puede dejar consigo serias consecuencias en el organismo de trabajadores relacionados con ambientes laborales saturado de este compuesto. Por ello, una de estas alteraciones es su gran adherencia al hierro presente en los grupos hemo de la hemoglobina, en la cual se halla un sitio núcleofilico con 240 - 250 de mayor afinidad que la unión que forma la hemoglobina con el oxígeno. Lo que conlleva a la muerte celular en algunos tejidos principales como miocardio (Cardiga et al., 2015) y nervioso (Arreguín et al., 2014). Tal situación, puede conllevar a la muerte del paciente, si a éste no se le presta la atención requerida.

Las condiciones laborales que relacionan los ambientes de los trabajadores de estaciones de servicio de gasolina requieren la determinación de las concentraciones de $\mathrm{CO}$, de tal manera que las mismas se controlen y de esta forma se eviten enfermedades relacionadas con esta exposición, para lo cual es necesario la implementación posterior de programa de prevención y promoción que ayuden a garantizar cada día una calidad de vida de éstos trabajadores de acuerdo a sus actividades laborales (Muñoz et al., 2007).

\section{Materiales y métodos}

La investigación, corresponde a un estudio de carácter descriptivo transversal, dentro de un enfoque cuantitativo. Donde se realizaron mediciones de las concentraciones de monóxidos de Carbono en los ambientes laborales de 9 estaciones de servicio de gasolina del municipio de Pitalito, con el monitor de gases portátil GX 2009, marca RKI que permite medir concentración de Oxigeno, $\mathrm{CO}, \mathrm{H}_{2} \mathrm{~S}$ e hidrocarburos alifáticos. Estas mediciones se realizaron cada 15 minutos en el transcurso de 2 horas en la mañana, el medio día, la tarde y la noche, repitiendo el procedimiento durante 3 jornadas de 8 horas laborales. Por lo tanto, se aplicó una encuesta a 57 trabajadores de las diferentes estaciones como parte del instrumento de recolección de datos y un consentimiento informado que garantice la participación voluntaria en el estudio en cuestión. Por otro lado, se utilizó la estación meteorológica RMW 200 de marca Oregón para determinar la presión atmosférica, temperatura, humedad relativa, velocidad del viento, dirección del viento en cada uno de los ambientes en cuestión.

Existen diversas metodologías que permiten la medición de monóxido de carbono, dentro de las cuales están presentes aquellas relacionadas con el monitoreo a partir de aparato portátil, que garantizan la 
cuantificación de la concentración de este gas en los ambientes laborales en tiempo real, como en el presente estudio. En algunos casos, se tiene en cuenta la medición de los porcentajes de carboxihemoglobina en sangre como bioindicador (Rojas et al., 2001). Y en término de medición global, se ha utilizado de forma frecuente la cromografía en fase gaseosa y la espectrometría que permite la obtención total de la concentración de oxígeno y el porcentaje de carboxihemoglobina en todo el torrente sanguíneo respectivamente.

\section{Resultados}

Las edades de los trabajadores de las estaciones, comprenden un promedio de 30.23 y una desviación estándar de $8.40(30,23 \pm 8,40)$, siendo los hombres el grupo mayoritario con $72 \%$. Al comparar las edades de las mujeres y los hombres, se evidenció que el valor-P es mayor que 0.05 , lo que indica que no existe una diferencia estadísticamente significativa entre las edades correspondientes (Tabla 1).

Tabla 1. Distribución de las edades en los trabajadores de las estaciones de servicio

\begin{tabular}{l|c|c|c|c|c|c|c}
\hline \multicolumn{1}{c|}{ Género } & $\boldsymbol{N}$ & $\boldsymbol{X}$ & $\boldsymbol{S D}$ & $\boldsymbol{C V}$ & Mín. & Máx. & Valor $\boldsymbol{P}$ \\
\hline F & 16 & 28,5 & 5,33 & $18,70 \%$ & 21,0 & 39,0 & \\
\hline M & 41 & 30,93 & 9,30 & $30,06 \%$ & 19,0 & 51,0 & \\
\hline Total & 57 & 30,23 & 8,40 & $27,77 \%$ & 19,0 & 51,0 & 0,33 \\
\hline
\end{tabular}

El desarrollo de esta investigación permitió la determinación de las concentraciones de monóxido de Carbono, a las cuales están expuestos los trabajadores de estaciones de servicio de la ciudad de Pitalito, durante las jornadas laborales, en sus quehaceres cotidianos, lo que corresponde a los promedios de las concentraciones de CO correspondientes en cada una de las ocho horas laborales. Como se aprecia en la Tabla 2 y en la Figura 1, en la primera estación se obtuvo un promedio de 37,20 ppm de CO con una desviación estándar de 19,47 $(37,20 \pm 19,47)$, en la segunda se obtuvo 48,82 ppm de CO y una desviación estándar de 25,82 (48,82 $\pm 25,82)$, en la tercera $(28,12 \pm 24,15)$, en la cuarta $(37,05 \pm 21,44)$, en la quinta $(10,77 \pm 12,56)$, la sexta $(30,47 \pm 25,05)$, en la séptima $(43,53 \pm 30,09)$, en la octava $(42,55 \pm 30,96)$, en la novena $(27,72 \pm 20,99)$. Es importante establecer que, aunque se identificaron algunos promedios por de bajos de los valores máximos permisibles durante una hora laborales; es considerable el hecho de que en algunos momentos de una hora laboral, éstos valores son exorbitantes y extremos, como en el caso 144 ppm y un mínimo de 0,00 ppm. La media de las concentraciones de $\mathrm{CO}$ durante una jornada laboral en las estaciones de servicio corresponde a 34,02 ppm. 
Tabla 2. Concentraciones de CO en las Estaciones de Servicio

\begin{tabular}{|c|c|c|c|c|c|c|c|c|c|}
\hline Estaciones & SID & ALC & LTP & LAB & LGE & Ств & ECS & SOL & MAR \\
\hline MA & 53,3 & 35,75 & 9,25 & 29 & 52,5 & 6 & 26 & 89,8 & 4,25 \\
\hline MA & 27 & 49,25 & 19,5 & 50,25 & 23,3 & 26,5 & 53,8 & 28,5 & 19,25 \\
\hline MA & 20,5 & 87,75 & 17 & 50,25 & 8,75 & 44 & 25 & 26,5 & 54,25 \\
\hline MA & 76 & 52 & 94,75 & 19,75 & 18 & 110 & 35,8 & 31,3 & 14,5 \\
\hline MA & 17,3 & 45,75 & 15,25 & 54,25 & 9,25 & 10,5 & 69,8 & 23,5 & 95,5 \\
\hline MA & 41,5 & 54 & 43,25 & 35,75 & 1,75 & 24,5 & 66,8 & 15,8 & 41,5 \\
\hline ME & 21 & 15,75 & 10,75 & 23,5 & 9,25 & 16,3 & 47 & 41,3 & 28,5 \\
\hline ME & 26,3 & 17,5 & 39,75 & 62,5 & 13,3 & 8,5 & 25 & 4,75 & 7,75 \\
\hline ME & 45,8 & 42,5 & 11,25 & 9 & 11,8 & 16,5 & 16,8 & 54 & 7,5 \\
\hline ME & 25,5 & 87,75 & 13,5 & 22,5 & 20,3 & 3,83 & 59,3 & 72,5 & 5,75 \\
\hline ME & 33,5 & 41 & 87 & 6,25 & 16,5 & 7,5 & 8,5 & 49,5 & 26 \\
\hline ME & 23,5 & 14,5 & 14,25 & 27 & 0 & 22,3 & 53,3 & 34,5 & 24,25 \\
\hline TA & 18,3 & 35,75 & 33,5 & 11,25 & 1,5 & 72,3 & 9 & 26,5 & 13,75 \\
\hline TA & 49,5 & 84,5 & 50,5 & 29 & 6,25 & 26,3 & 29,5 & 49,5 & 43 \\
\hline TA & 42,8 & 57,25 & 6,75 & 54,5 & 1,75 & 17,8 & 42,3 & 25,3 & 28,5 \\
\hline TA & 91,3 & 49,5 & 15,75 & 54,25 & 4,5 & 12 & 70,3 & 42,3 & 36,75 \\
\hline TA & 45,3 & 59,75 & 30 & 17,5 & 4 & 49 & 21 & 88 & 24,25 \\
\hline TA & 65 & 37,75 & 17,25 & 82,25 & 36,3 & 26,5 & 18 & 52 & 52,75 \\
\hline NO & 21 & 16,25 & 16,25 & 45 & 2,25 & 55,8 & 20 & 37,3 & 24,5 \\
\hline NO & 15,8 & 9,5 & 60,75 & 42,75 & 0 & 22,5 & 81,8 & 41,3 & 27,75 \\
\hline NO & 22,3 & 101,3 & 35 & 10 & 2 & 12 & 104 & 23,8 & 51 \\
\hline NO & 45 & 78,25 & 5,25 & 41,75 & 0 & 35 & 122 & 144 & 15,75 \\
\hline NO & 36,8 & 29,75 & 16,5 & 82 & 11,3 & 56,3 & 15,3 & 8,25 & 7,5 \\
\hline NO & 29,3 & 68,75 & 12 & 29 & 4,25 & 50 & 25,5 & 11,5 & 10,75 \\
\hline
\end{tabular}

MA (Mañana), ME (Medio Día), TA (Tarde), NO (Noche) 


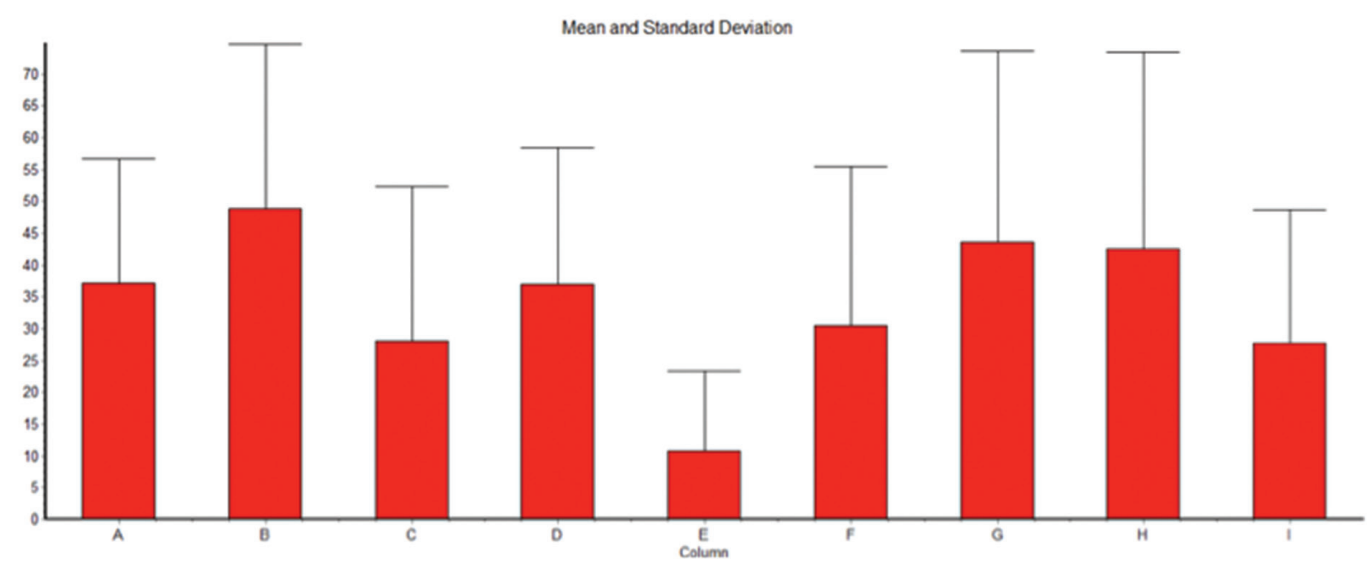

Figura 1. Desviación estándar de concentraciones de $\mathrm{CO}$ en las estaciones de servicio Estaciones SID (A), ALC (B), LTP (C), LAB (D), LGE (E), CTB (F), ECS (G),SOL (H), MAR (I).

Después del procesamiento de los datos correspondientes a los promedios de cada una de las ocho horas, durante tres jornadas en las estaciones de servicio participantes, se aplicó una prueba de normalidad (Tabla 3), lo que evidenció que el sesgo estandarizado y la curtosis estandarizada se encuentran fuera del rango de $-2 \mathrm{a}+2$ en siete de las nueve estaciones de servicio. Esto indica, que los datos obtenidos en estas muestras no presentan un comportamiento de distribución normal. Lo anterior, permite la comparación de las medianas en lugar de los promedios de las concentraciones de CO en cada estación, por medio de la prueba de Kruskal-Wallis, la cual expreso un valor de $P=2,54$ dentro de un nivel de confianza del $95 \%$. Esta situación, confirma que las medianas de las concentraciones de monóxidos de carbono en cada estación, difieren considerables y presentan una divergencia estadísticamente significativa a la hora de asumirla como una población total.

Tabla 3. Prueba de normalidad. Concentraciones de CO en las estaciones de servicio

\begin{tabular}{|c|c|c|c|c|c|c|c|c|c|c|}
\hline Est. & $N$ & $x$ & $S D$ & $\mathrm{CV}$ & Mín. & Máx. & Rango & Sesgo & Curtosis & $\begin{array}{c}\text { Kruskal- } \\
\text { Wallis. }\end{array}$ \\
\hline SID & 24 & 37,20 & 19,48 & $52,35 \%$ & 15,75 & 91,25 & 75,5 & 2,53 & 1,41 & 125,89 \\
\hline ALC & 24 & 48,82 & 25,83 & $52,9 \%$ & 9,5 & 101,25 & 91,75 & 0,69 & $-0,65$ & 146,20 \\
\hline LTP & 24 & 28,12 & 24,15 & $85,87 \%$ & 5,25 & 94,75 & 89,5 & 3,31 & 2,25 & 90,65 \\
\hline LAB & 24 & 37,05 & 21,45 & $57,89 \%$ & 6,25 & 82,25 & 76,0 & 1,07 & $-0,27$ & 123,70 \\
\hline LGE & 24 & 10,77 & 12,57 & $116,66 \%$ & 0 & 52,5 & 52,5 & 4,03 & 4,65 & 38,52 \\
\hline СТВ & 24 & 30,47 & 25,05 & $82,22 \%$ & 3,83 & 109,75 & 105,92 & 3,23 & 3,096 & 99,0 \\
\hline ECS & 24 & 43,53 & 30,09 & $69,12 \%$ & 8,5 & 121,5 & 113,0 & 2,13 & 0,65 & 129,97 \\
\hline SOL & 24 & 42,55 & 30,96 & $72,75 \%$ & 4,75 & 144,0 & 139,25 & 3,51 & 4,03 & 128,18 \\
\hline MAR & 24 & 27,71 & 20,99 & $75,72 \%$ & 4,25 & 95,5 & 91,25 & 3,13 & 3,43 & 94,354 \\
\hline Total & 216 & 34,02 & 25,86 & $76,01 \%$ & 0 & 144,0 & 144,0 & 7,39 & 4,98 & 125,89 \\
\hline
\end{tabular}


Lo anterior, permite considerar a la población en estudio, como un fenómeno de distribución particular, que obedece a la aplicación de pruebas no paramétricas. Dentro de las cuales, está presente la correlación de Spearman rho, utilizada para relacionar algunas variables. Para éste caso, se asociaron las concentraciones de monóxido de Carbono con las temperaturas medidas en los ambientes laborales (Tabla 4 y Figura 2), obteniendo un coeficiente de correlación de $(-0,024)$, que demuestra que no existe relación significativa entre estas dos variables y por ende que la temperatura no incide de forma determinante en la saturación del $\mathrm{CO}$ presentes estos ambientes. Caso similar, sucede al asociar las concentraciones de $\mathrm{CO}$ con la Humedad relativa, con un coeficiente rho de 0,028 . Las concentraciones de los compuestos en niveles de explosividad (LEL) corresponden a $(0,73 \pm 1,43)$, lo que demuestra un riesgo insignificante con respecto a la exposición de los trabajadores.

Tabla 4. Concentraciones de CO relacionadas con los síntomas de algunos trabajadores

\begin{tabular}{l|c|c|c|c|c|c|}
\hline Síntomas & $\boldsymbol{N}$ & $\boldsymbol{X}$ & $\boldsymbol{S D}$ & Mín. & Máx. & Valor-P \\
\hline Alteraciones Visuales & 5 & 40,56 & 5,17 & 37,05 & 48,82 & \\
\hline Cefalea & 20 & 32,09 & 8,97 & 10,77 & 43,53 & \\
\hline Debilidad Muscular & 3 & 41,09 & 3,41 & 37,2 & 43,53 & \\
\hline Dolor Abdominal & 3 & 32,28 & 18,64 & 10,77 & 43,53 & \\
\hline Dolor Torácico & 3 & 40,77 & 3,09 & 37,2 & 42,55 & \\
\hline Nausea Vomito & 4 & 31,86 & 14,29 & 10,77 & 42,55 & \\
\hline Ninguna & 16 & 33,01 & 11,04 & 10,77 & 48,82 & \\
\hline Vértigo & 3 & 40,02 & 10,98 & 27,71 & 48,82 & \\
\hline Total & 57 & 34,43 & 10,15 & 10,77 & 48,82 & $\mathbf{0 , 4 5}$ \\
\hline
\end{tabular}

Gráfico Caja y Bigotes

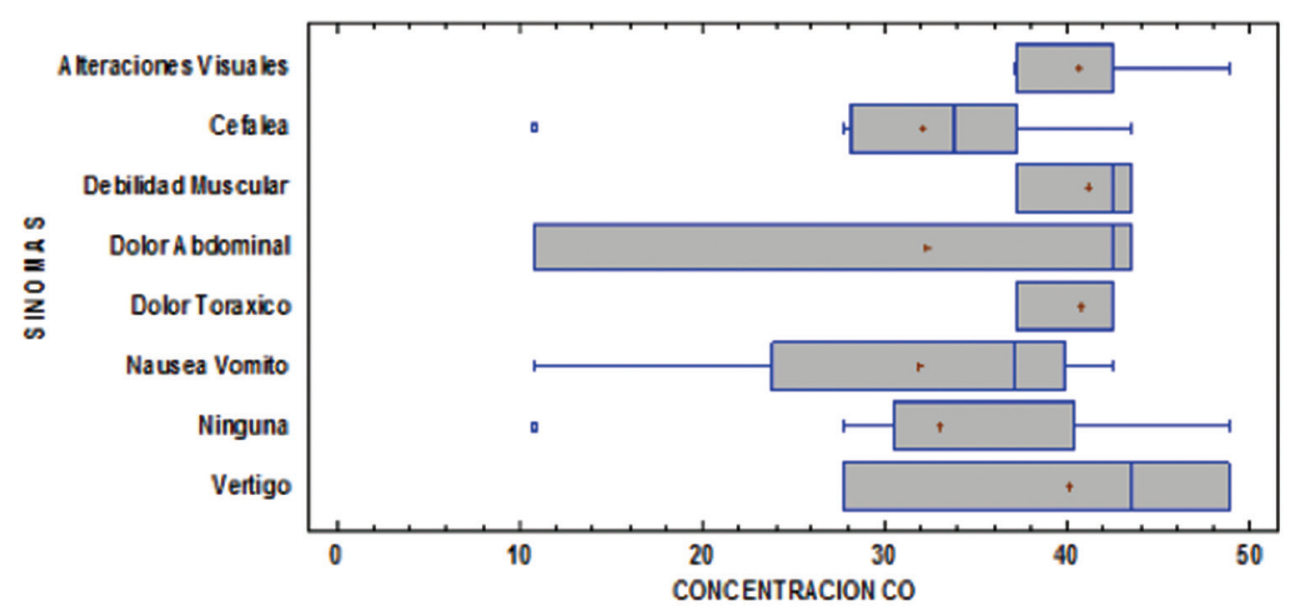

Figura 2. Desviación estándar de concentraciones de CO relacionadas con los síntomas de algunos trabajadores 
De acuerdo a las manifestaciones expresadas por cada uno de los trabajadores participantes, se obtuvo que la cefalea es el síntoma de mayor frecuencia, mientras que la debilidad muscular, dolores abdominales y vértigo son los de menor predominancia; aunque las alteraciones visuales, debilidad muscular, dolor torácico y vértigo se han presentado en trabajadores expuestos a mayores concentraciones de $\mathrm{CO}$ en sus ambientes laborales, todas alrededor de $40 \mathrm{ppm}$ aproximadamente. Por otro lado, el valor de $P$ es 0.45 , indica que no existe una diferencia estadísticamente significativa de las concentraciones de $\mathrm{CO}$ de los ambientes laborales de los trabajadores que manifestaron los síntomas correspondientes. Esta situación deja en manifiesto, que los síntomas manifestados por los trabajadores no siguen un patrón proporcional a las concentraciones de $\mathrm{CO}$ presentes en estos ambientes laborales.

\section{Discusión}

Según Donati (2015), la organización mundial de la salud (OMS), determinó que los limites máximo permisibles (TLW) de exposición al CO es de 10ppm durante hora 8horas y $90 \mathrm{ppm}$ de $\mathrm{CO}$ durante 15 minutos; aunque la exposición a $\mathrm{CO}$ en las diferentes estaciones de servicio no fue continua, es muy importante establecer que estas concentraciones, en muchos casos, sobrepasaron los límites máximo permisibles y en ocasiones excedieron de forma exuberante tales niveles, como en el caso de 114 ppm durante 15 minutos en una de la estaciones. Tal situación, conlleva a establecer medidas de prevención y promoción, debido a que la exposición al CO es crónica con un promedio de 54 meses de tiempo laborado, donde un trabajador puede llegar a trabajar hasta 30 turnos de 8 horas en un mes.

Algunas de las manifestaciones clínicas de la exposición al CO están relacionadas con la cefalea, aunque este síntoma puede tener diferentes causas, en el presente estudio cerca del $35 \%$ de los trabajadores manifestaron haber presentado muy frecuente este trastorno. Situación que se agudiza, al evidenciar que el promedio de exposición a $\mathrm{CO}$ de estos trabajadores corresponde a 32,09 ppm en cualquiera en una hora laboral. De acuerdo a lo anterior, es coherente establecer que la cefalea es el síntoma más frecuente en trabajadores expuestos al CO como lo referencian muchos autores.

\section{Conclusiones}

Aunque las concentraciones de CO no son continuas, en muchos casos están muy por encima de los TLW, lo que puede estar relacionado con los diferentes síntomas presentados por los trabajados de las estaciones de servicio.

Las concentraciones de $\mathrm{CO}$ de las estaciones de servicio del municipio de Pitalito no están influenciadas en relación directamente proporcional por la temperatura y la humedad, es decir, la saturación de $\mathrm{CO}$ es independiente de estas dos variables.

En la mayoría de los casos, las concentraciones de $\mathrm{CO}$ no siguen un patrón de relación directamente proporcional con los síntomas manifestados por los trabajadores; pero muchas de están alteraciones pueden estar directamente relacionas con el tiempo de exposición al CO.

\section{Agradecimientos}

Los autores expresan sus agradecimientos a Julián Castañeda de UNIMINUTO por su apoyo incondicional, a los estudiantes del grupo OCUPAMED y a los trabajadores de las estaciones de servicio de Pitalito, participantes en la investigación.

\section{Literatuta Citada}

1. Arreguín-González, I. J., Ayala-Guerrero, F., GascaZamudio, P. D. \& Melo-Balderas, M. A. (2014). Funciones neurocognitivas alteradas en paciente intoxicada por monóxido de carbono. Neurología, Volume 31, Issue 6, Pages 416-417 http://dx.doi.org/10.1016/j. nrl.2014.11.004.

2. Cardiga, R., Proenca, M., Carvalho, C., Costa, L., Botella, A., Marques, F., Paulino, C., Carvalho, A. \& Fonseca, C. (2015). Intoxicacão por monóxido de carbono com compromisso cardíaco: o que sabemos?. Rev Port Cardiol. 34(9):557-560. 
3. Castillo, D. \& Casan, P. (2006). Monóxido de carbono: Dos caras de un mismo personaje. Arch Bronconeumol. 42(10):489-9.

4. Donati, S., Gainnier, M. \& Chibane-Donati, O. (2015). Intoxicación por monóxido de carbono. EMC. 36(10):1-17.

5. Fleta, J., Fons, C., Arnauda, P., Ferrer, A. \& Olivares, J. L. (2005). Intoxicación por monóxido de carbono. An Pediatr (Barc). 62(6):587-90.

6. Guzmán, J. A. (2012). Carbon Monoxide Poisoning. Critical Care Clinics. 28(4): 537-548.

7. Hampson, N. B. (2016). Cost of accidental carbon monoxide poisoning: A preventable expense. Preventive Medicine Reports. (3):21-24.

8. Hampson, NB. \& Bodwin, D. (2013). Toxic CO-ingestions in Intentional Carbon Monoxide Poisoning. The Journal of Emergency Medicine. 44(3): 625-630.

9. Oliu, G., Nogué, S. \& Miró, O. (2010). Intoxicación por monóxido de carbono: claves fisiopatológicas para un buen tratamiento. Emergencias. (22): 451-459.

10. Rojas, M., Dueñas, A. \& Sidorovas, L. (2001). Evaluación de la exposición al monóxido de carbono en vendedores de quioscos. Valencia, Venezuela. Rev Panam Salud Publica/Pan Am J Public Health. 9(4): 240-245.
11. Romero Placeres, M., Diego Olite, F. \& Álvarez Toste, M. (2006). La contaminación del aire: su repercusión como problema de salud. Rev Cubana Hig Epidemiol. 44(2): $1-14$

12. Sandilands, E. A. \& Bateman, D. N. (2016). Carbon monoxide. Medicine. 44(3):151-152.

13. Sircar, K., Clower, J., Shin M., Bailey, C., King, M. \& Yip, F. (2015). Carbon monoxide poisoning deaths in the United States, 1999 to 2012. The American Journal of Emergency Medicine. 33(9): 1140-1145.

14. Téllez, Jairo, Rodríguez, Alba \& Fajardo, Álvaro. (2006) Contaminación por Monóxido de Carbono: un Problema de Salud Ambiental. Rev. salud pública. 8 (1): 108117.

15. Muñoz, A. M., Paz, J. J. \& Quiroz, C. M. (2007). Efectos de la contaminación atmosférica sobre la salud en adultos que laboran a diferentes niveles de exposición. Rev. Fac. Nac. Salud Pública. 25(2): 85-94.\}

16. Vargas, T. (2006). Toxicología. Capítulo 83. Toxicidad por Monóxido de Carbono. 582-586. Grupo Difusión. Recuperado de: http://www.geocities.ws/lucho16/documents/Monoxido_Carbono_L_Vargas.pdf
Conflicto de Intereses

Los autores declaran no tener ningún conflicto de intereses

Recibido: 20 de octubre de 2015 Aceptado: 24 de noviembre de 2015 